\title{
Slot Antenna in Ridge Gap Waveguide Technology
}

\author{
Ashraf Uz Zaman \\ Department of Signals and System \\ Chalmers University of Technology \\ SE- 41296, Göteborg, Sweden \\ zaman@chalmers.se
}

\author{
Per-Simon Kildal \\ Department of Signals and System \\ Chalmers University of Technology \\ SE-41296, Göteborg, Sweden \\ per-simon.kildal@chalmers.se
}

\begin{abstract}
A Slot antenna design based on recently developed gap waveguide technology has been presented in this work. This antenna can be built easily by having the feed network on the bottom metal plate, where the ridge will be distributing the waves for equal excitation of each slot element, and the top metal plate will have the slots. Design and simulation results of the basic $T$ junction for a corporate-feed network and the single element slot show that- it is possible to have an array antenna with BW of 15 $\%$ or more based on ridge gap waveguide technology.
\end{abstract}

\section{Keywords- Slot array antenna, corporate-feed, T-junction}

\section{INTRODUCTION}

Planer waveguide slot array antennas and microstrip patch array antennas have been an interesting research topic among the antenna professionals since 1970s. Typical printed antennas can be compact, lightweight and can offer low cost but these types of antennas suffer usually from high dielectric losses at high frequencies. Also, when an array of printed antenna is built for high gain or multiple beam application, the antenna efficiency decreases even more because of increased losses in feeding network. The steps and discontinuities in the feeding network also tend to give spurious radiation [1]. Planar waveguide-type slot array antennas do not suffer from the above disadvantages, and is therefore an attractive candidate for high frequency applications requiring high gain [2-4]. But waveguide slot arrays are associated with complex feed network under the radiating waveguide array and high manufacturing costs of standard rectangular waveguide at high frequency. Apart from the cost of waveguide array itself, the integration of high frequency MMIC with rectangular waveguide is also very challenging at high frequencies [5-6].

Recently evolved gap waveguide technology described and verified in [7-9] can be applied very successfully to address some of these problems. The low loss feed network needed for an array can be built very easily with the help of ridge gap waveguide concept. In addition, radiating slots can be placed conveniently on the top smooth metal plate of ridge gap waveguide. Thus, the ridge gapwaveguide slot antenna can be an attractive as well as cost effective solution for high gain and high efficiency applications.

There has till now not been any publications on gap waveguide antennas, except for the horn antenna in [10], and the initial works in single-hard wall waveguide slot arrays in [11]. Therefore, the purpose of the present paper is to present the first ridge gap waveguide excited slot antenna. We consider a fully radiating single slot, i.e. a slot from which all the incident power is radiated, corresponding to using a fully branched distribution network, i.e. a corporate-feed network. We also present a single T-junction for use together with this slot in the corporate-feed network. The final conference paper will also show results for a $2 \times 2$ array using the slot and $\mathrm{T}$ junction design in this short abstract

\section{DESIGN OF T-JUNCTION For CORPORATE FEED}

The basic component of a usual array antenna corporatefeed network is a T-junction. The T-junction usually consists of a quarter wave transformer section and three $50 \Omega$ lines. This is shown in figure 1 . The simulation results for the basic Tjunction is shown in figure 2 . The simulation results show that it will be possible to design a wideband feed network for a complete array antenna based on this T-junction.

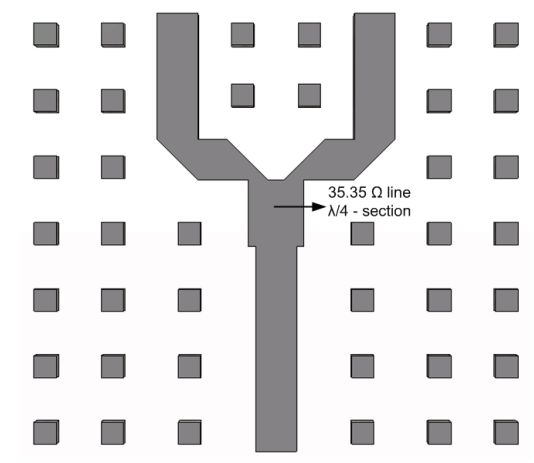

Figure 1. Single T-junction for corporate feed network (Top metal plate not shown)

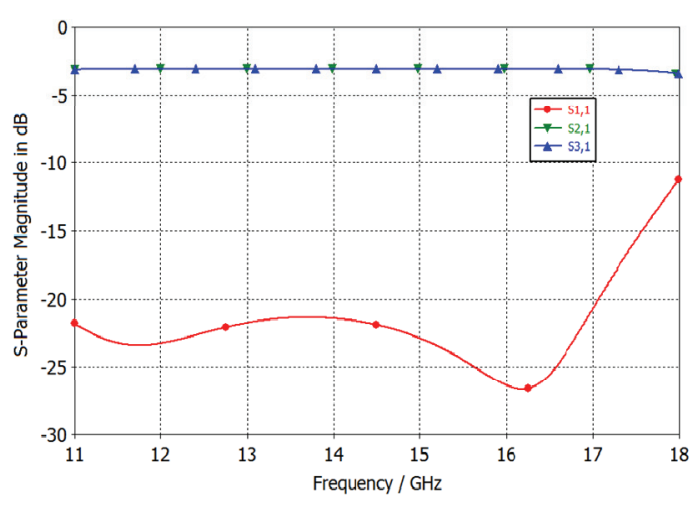

Figure 2. Simulation results for the T-junction

The work has been funded by Swedish Research Council VR 


\section{Single Slot DESIGN}

The slot array antenna design usually starts with a single element slot. Similar approach is followed in this work as well. The single slot antenna excited by ridge gapwaveguide is shown in figure 3 . The single element is designed to operate in $12-14 \mathrm{GHz}$ frequency range. The length and the width of the slot element is varied to have the return loss around $15 \mathrm{~dB}$ over the above mentioned bandwidth. The reflection coefficient for the single element is shown in figure 4.

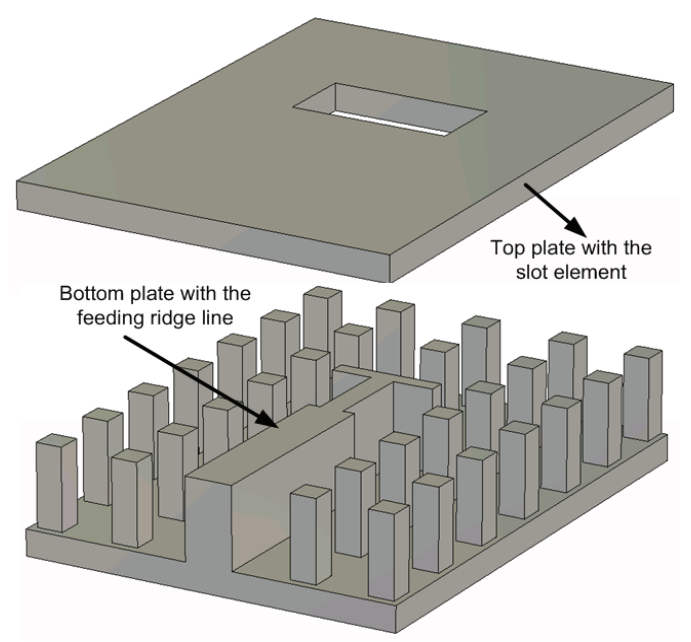

Figure 3. Single element slot in gapwaveguide technology

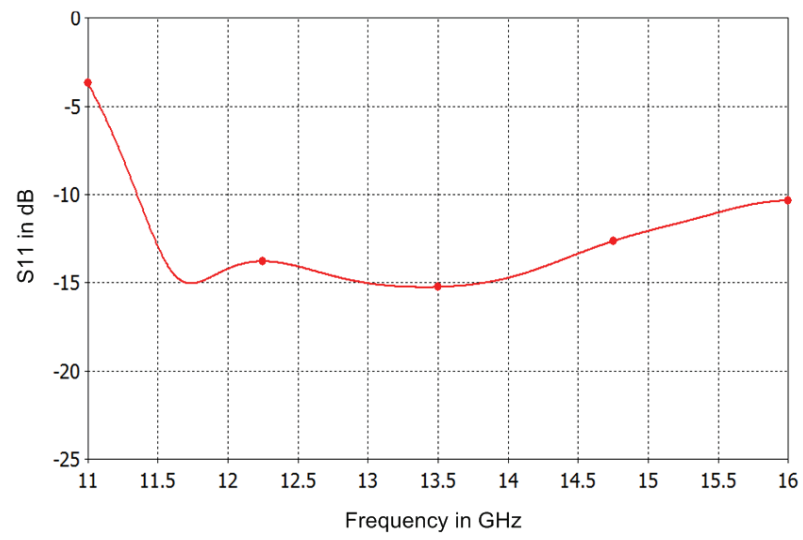

Figure 4 . Single element slot in gapwaveguide technology

\section{CONCLUSION}

We have presented a slot antenna design based on ridge gapwaveguide technology. A single T-junction is designed as a basic unit of the entire corporate-feed network. Also a single element slot antenna is designed by having a slot on the top metal plate of the ridge gapwaveguide. Simulated results clarify that - both the T-junction and the slot have reasonably wide bandwidth, and it will be possible to use these basic elements for the entire array design.

\section{REFERENCES}

[1] E.Levine, G. Malamud, S.Shtrikman and D. Treves, "A study of microstrip array antennas with the feed network", IEEE Transection on Antennas and Propagation, vol.37, no.4, pp.426-464, April 1989.

[2] J. Hirokawa and M. Ando, "Single-layer feed waveguide consisting of posts for plane TEM wave excitation in parallel plates", IEEE Trans. Antennas Propag., vol. 46, no. 5, pp. 625-630, May 1998.
[3] J. Hirokawa and M. Ando, "Efficiency of $76 \mathrm{GHz}$ Post-wall Waveguidefed Parallel-plate Slot Arrays", IEEE Trans. Antennas Propag., vol. 48 no. 11, pp. 1742-1745, November 2000

[4] M. Ohira, A. Miura, M. Ueba, " $60 \mathrm{GHz}$ Wideband Substrate-IntegratedWaveguide Slot Array Using closely Spaced Elements for Planer Multisector Antenna", IEEE Trans. Antennas Propag., vol. 58, no. 3, pp. 993-998, March 2010

[5] V. Vassilev, N. Wadefalk, R.Kozhuharov, M. Abbasi, S.E Gunnarsson, H.Zirath, T. Pellikka, A. Emrich, M. Pantaleev, I.Kallfass, A. Leuther, "MMIC based components for MM-Wave Instrumentaion," IEEE Microwave and Wireless Components Letters, vol. 20, no.10, pp. 578580, October 2010.

[6] A.U Zaman, V.Vassilev, P.S Kildal, A. Kishk,"Increasing Parallel Plate Stop-band in Gap Waveguides using Inverted Pyramid-Shaped Nails for Slot Array Application Above 60GHz", European Conference on Antennas and Propagation, Rome, April 2011.

[7] P.-S. Kildal, E. Alfonso, A. Valero-Nogueira, E. Rajo-Iglesias, "Local metamaterial-based waveguides in gaps between parallel metal plates", IEEE Antennas and Wireless Propagation letters, Volume 8, pp. 84-87, 2009.

[8] P.-S. Kildal, A. Uz Zaman, E. Rajo-Iglesias, E. Alfonso and A. ValeroNogueira, "Design and experimental verification of ridge gap waveguides in bed of nails for parallel plate mode suppression", IET Microwaves, Antennas \& Propagation, Vol.5, Iss.3, pp. 262-270, March 2011.

[9] M. Bosiljevac, Z. Sipus, P.-S. Kildal, "Construction of Green's functions of parallel plates with periodic texture with application to gap waveguides - A plane wave spectral domain approach", IET Microw. Antennas Propag., Vol. 4, Iss. 11, pp. 1799-1810, Nov. 2010

[10] Ahmed Kishk and P.-S. Kildal, "Quasi-TEM H-plane Horns with Wideband Open Hard Side-Walls," 4th European Conference on Antennas and Propagation, 2010, Barcelona, Spain, April 12-16, 2010.

[11] A. Valero- Nogueira, E. Alfonso, J.I. Herranz and M. Baquero, “ Planar slot array antenna fed by oversized Quasi TEM waveguide", Microwave and Optical Technology Letters, Vol. 49, no 8, pp.1875-1877, 2007. 